\title{
Point-of-Care Ultrasound Reduces Visit Time and Cost of Care for Infants with Developmental Dysplasia of the Hip
}

\author{
Christina Herrero $^{1}$ (D) $\cdot$ Yhan Colon ${ }^{2} \cdot$ Akash Nagapurkar $^{3} \cdot$ Pablo Castañeda $^{1}$
}

Received: 16 August 2021 / Accepted: 1 October 2021 / Published online: 19 October 2021

(c) Indian Orthopaedics Association 2021

\begin{abstract}
Background Healthcare institutions and policymakers are searching for system-wide approaches to reduce costs while maintaining quality and improving patient outcomes. In most healthcare systems infants referred for the detecting or treating developmental dysplasia of the hip (DDH) are sent to a radiology department for sonographic evaluation. The total duration of visit and cost of visit are essential variables in any healthcare setting and affect both efficiency and "the bottom line". By having the treating clinician perform point-of-care ultrasound (POCUS) for the detection and follow-up of patients with $\mathrm{DDH}$, we hypothesize that there would be a significant reduction in the time spent on the visit and the cost incurred without compromising quality or patient satisfaction. To our knowledge, no prior study has examined the effect of incorporating POCUS on the duration and cost of the visit in patients with DDH.

Purpose To determine if there was a difference in the duration of the visit for patients with DDH when POCUS was performed compared to when traditional "formal" sonography was performed. To determine if there was a difference in the cost of the visit for patients with DDH when POCUS was performed compared to when traditional "formal" sonography was performed.

Methods Data for visits to a specialized outpatient office were collected over two years at a single-specialty orthopedic hospital, comparing the duration and cost of the visit between patient encounters for infants who had "formal" sonograms performed in the radiology suite to infants who underwent POCUS of the hip. In all, we included 532 patient encounters, 326 patients had POCUS performed, and 206 had a "formal" ultrasonographic evaluation performed. Of these, 140 were new evaluations and 392 were follow-up evaluations for treatment. Of the 140 new patients, 80 were in the POCUS group, and 60 were in the "formal" US group. Of the 392 follow-ups, 246 were in the POCUS group, and 146 were in the "formal" US group.

Results The mean duration of the encounter for the POCUS group was 42 min (range 16-75 min), and for the "formal" US group, it was $92 \mathrm{~min}$ (range 36-163 min). This difference was statistically significant $(p=0.002)$. The mean cost of the encounter for the POCUS group was $\$ 121.13$, and for the "formal" US group, it was $\$ 339.38$. This difference was statistically significant $(p=0.002)$.

Conclusion Ultimately, our study demonstrated a statistically significant reduction in the duration and cost of a patient encounter for infants with DDH when they undergo POCUS rather than "formal" sonographic evaluation.
\end{abstract}

Keywords Developmental dysplasia of the hip · Point of case ultrasound · Pediatric orthopaedics

\section{Introduction}

Christina Herrero

christinapherrero@gmail.com

1 Department of Orthopaedic Surgery, NYU Langone Health, New York, NY, USA

2 Icahn School of Medicine at Mount Sinai, New York, NY, USA

3 New York University, New York, NY, USA
Developmental dysplasia of the hip (DDH) is the most common pediatric orthopedic condition in newborns. Though it is common worldwide, there are significant differences in its reported incidence, which may function from the number of detected cases rather than an actual incidence. A classic study cited an incidence of $5.5 \%$ in a cohort of 18,060 newborns but acknowledged that reported rates range from 4.4 to $51.8 \%$ [1]. 
There is wide variability in screening methods, which has led to uncertainty in the accuracy of reported incidence. In more recent literature in which ultrasound (US) has become the most common screening method, there are still significant inconsistencies in incidence, ranging from 1 to 175 per 1000 live births [2-5]. Inconsistencies are essential to understand and address as undiagnosed DDH leads to accelerated hip joint degeneration, necessitating total hip arthroplasty earlier in life $[6,7]$.

Several countries around the world have successfully implemented national screening to address inconsistencies in screening and late diagnoses. Since 1992 in Austria and 1996 in Germany, universal ultrasound screening programs have significantly reduced the number and severity of surgical interventions related to DDH [8]. Though universal screening programs have been cost-effective in preventing future, more invasive and expensive care, they have also led to added fees with additional visits. In more recent years, several countries have incorporated point-of-care ultrasound (POCUS) into their screening programs to combine the benefits of universal screening with the cost reduction and increased feasibility of POCUS.

Healthcare delivery systems are complex and do not allow easy and universal access to ultrasonography or radiography. Often clinicians are unaware of the lowering cost and efficiency of incorporating POCUS into their practices.

POCUS has dramatically expanded the possibilities of screening in both hospital and outpatient settings and streamlined the process of diagnosing patients. Though this ultrasound exam is different from that for DDH, the capability of the treating physician to perform it in the office versus sending the patient to a radiology suite for a formal US is relevant and can save time. Often clinicians are unaware of the lowering cost and efficiency of incorporating POCUS into their practices.

In light of the growing literature surrounding the improvement of DDH screening and POCUS use, we wanted to investigate the effects POCUS had on the time and cost of visits for DDH. There were two purposes of this study. The first purpose was to determine if there was a difference in the encounter duration for patients with DDH when POCUS was performed compared to when traditional "formal" sonography was performed. The second purpose was to determine if there was a difference in the cost of the encounter for patients with DDH when POCUS was utilized compared to when traditional "formal" sonography was obtained.

\section{Methods}

\section{Study Design and Setting}

We performed a retrospective review of prospectively collected data to assess whether the duration and cost of the patient encounter for a primary diagnosis of DDH differed when POCUS was incorporated than when a "formal" ultrasonographic examination was obtained.

The primary endpoints were the duration and cost of the encounter. The duration of the encounter was determined by analyzing the electronic time stamp created in the electronic medical record (EMR) from the time the patient checked in to the time they checked out. We determined the overall cost of the encounter was determined by the level of service and any additional fee for service recorded in the EMR.

Over two years, we conducted the study in an outpatient setting in a subspecialty pediatric orthopedic practice where one surgeon performed POCUS and three others obtained "formal" ultrasound evaluations.

\section{Patients}

The cohort was composed of a series of 532 patient encounters for patients younger than ten months presenting to a specialty pediatric orthopedic practice for a hip evaluation. There were 140 new evaluations, referred because they had a positive or equivocal physical examination result or were at a high risk of having DDH, and 392 follow-up encounters for treatment. Of the entire cohort, 326 patients had POCUS performed, and 206 had a "formal" ultrasonographic evaluation performed. We only included patients in the "formal" ultrasonographic group when the evaluation was performed on the same day and counted as part of the same encounter. All patients who had ultrasound performed on a different day were excluded from this study in order to accurately include all time associated with the entirety of the visit.

Of the 140 new patients, 80 were in the POCUS group, and 60 were in the "formal" US group. Of the 392 followups, 246 were in the POCUS group, and 146 were in the "formal" US group.

\section{Statistical Analysis}

We analyzed data on SAS software. We utilized a two-sampled independent $t$ test to compare the means of the normally distributed interval dependent variables for the two groups and a Wilcoxon-Mann-Whitney $U$ test as a non-parametric analog to the independent $t$ test when it was assumed that the dependent variable was a normally distributed interval. Statistical significance was set at a $p$ value less than 0.05 .

\section{Ethical Approval}

We obtained ethical approval for this study from the New York University School of Medicine (study number i170096_MOD09). The parents of the included patients were informed of the study, and written consent was obtained. 


\section{POCUS Examination}

For the POCUS, a portable US was used that the provider can carry and plug into a smartphone device or tablet. This portable US was available at all times in the clinic. Once the patient's MRN was entered, the images were synced with the patient's chart, and all were subsequently uploaded into the electronic medical record system. The primary surgeon performing the exam is a fellowship-trained pediatric orthopedic surgeon. The exam, executed by the primary surgeon consisted of images of the femoral head in the acetabulum to evaluate the degree of reduction, subluxation or displacement; a dynamic image with adduction, flexion and a posteriorly directed force to evaluate stability and finally an image of the femoral head in the acetabulum to measure the alpha and beta angles. All images were of high quality, equivalent to those from traditional US evaluations. The POCUS took five minutes-including preparing the examination table, positioning the infant, logging the information, the exam itself and saving the pictures.

\section{Flow of Clinic Visits}

In the health care setting where this study was carried out, patients are checked into an electronic medical record system at the time they arrive for their visit; this creates a timestamp which is reflected in the patients chart. Similarly when the patient checks out at the end, a timestamp is created noting that the visit has ended; for the purposes of this study, we utilized the time at check in and the time at final checkout to calculate the duration of the visit. For patients in the "formal" ultrasound group, some would be evaluated clinically, then sent for ultrasound in the radiology suite and then return for the clinician to interpret the results, others would go first to radiology and then to the clinic. The specific order of these events was not recorded. For the POCUS group, the ultrasound occurred after the physician examined the infant and spoke with the caregiver. Again, the specific order of these events was not recorded (Fig. 1).

\section{Costs}

Cost information was obtained from our billing office. (Table 1) We averaged all charges for formal US and POCUS for both the initial and follow-up visits. The amounts are the total fees that were billed to the patient's insurance. There were no Medicaid patients, all patients had some form of private health insurance.

\section{Results}

The mean age of the patients was 4.4 months (range $0.25-9.5$ months, or $2-42$ weeks). There were no significant differences in the groups' demographics, with the mean age of the POCUS group being 4.5 months (range $0.25-9.5$ months) and the mean age of the "formal" US group being 4.25 (range $0.25-6.25$ months) $(p=0.6$ ).

The mean duration of the encounter for the POCUS group was $42 \mathrm{~min}$ (range 16-75 $\mathrm{min}$ ), and for the "formal" US group, it was $92 \mathrm{~min}$ (range 36-163 min). This difference was statistically significant $(p=0.002)$.

The mean cost of the encounter for the POCUS group was $\$ 121.13$, and for the "formal" US group, it was $\$ 339.38$. This difference was statistically significant $(p=0.002)$.

When broken down by whether it was a new or followup encounter: the cost for a new patient encounter in the POCUS group was $\$ 162.35$ and $\$ 370.63$ for the "formal" US group. The cost for a follow-up encounter in the POCUS group was $\$ 107.72$ and $\$ 333.90$ for the "formal" US group. This difference was found to be statistically significant with a $p$ value of 0.003 (Table 1). This demonstrated there was significant money saved for both new patient and follow-up encounters.

\section{Traditional US vs. Point of Care US Patient Visit Flow Chart}
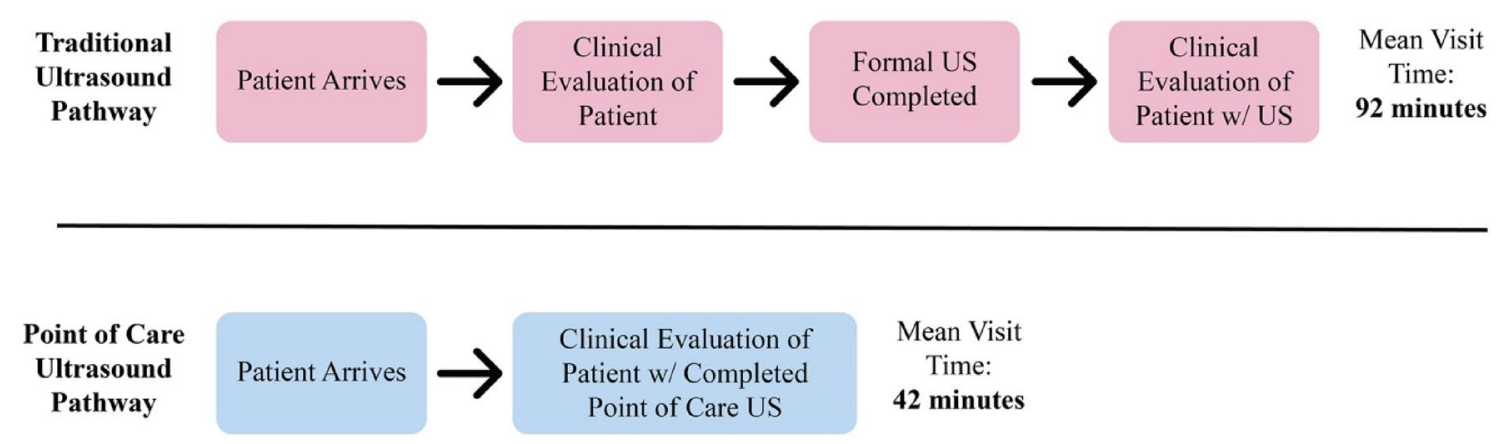

Fig. 1 Components of clinic visit flowchart 
Table 1 Cost breakdown of costs by cohort and patient visits

\begin{tabular}{|c|c|c|c|c|}
\hline & \multicolumn{2}{|c|}{ Formal ultrasound cohort } & \multicolumn{2}{|c|}{ Point-of-care ultrasound cohort } \\
\hline & New patient visit & Follow-up visit & New patient visit & Follow-up visit \\
\hline & $N=60(29 \%)$ & $N=146(71 \%)$ & $N=80(25 \%)$ & $N=246(75 \%)$ \\
\hline $\begin{array}{l}\text { Mean cost per visit (Surgeon } \\
\text { encounter only)* }\end{array}$ & USD \$113.64 & USD \$76.16 & USD \$162.36 & USD \$107.72 \\
\hline Formal ultrasound additional cost & USD $\$ 257.32$ & USD $\$ 257.32$ & - & - \\
\hline Mean cost per encounter & USD $\$ 370.96$ & USD $\$ 333.90$ & USD \$162.36 & USD \$107.72 \\
\hline
\end{tabular}

*For the Point-of-Care Ultrasound (POCUS) Cohort, the hospital bills the visit with the surgeon at a slightly different rate to account for the POCUS being conducted

\section{Discussion}

Our study demonstrated a significant reduction in the duration and cost of a patient encounter for infants with DDH when they undergo POCUS rather than "formal" sonographic evaluation. To our knowledge, this is the first study that demonstrates the cost and time reduction for POCUS for the pediatric orthopedic population and has significant implications across all fields of medicine.

POCUS will increase our ability to screen for DDH by making it easier, faster, and less expensive for families to evaluate their children. We also know that early identification and introduction of treatment can significantly alter the subsequent invasive procedures and associated complications that are historically associated with DDH [9]. If there is a clinical concern for DDH, most physicians have traditionally ordered an ultrasound. The patients then have to schedule this test, complete it, very often in a different facility or part of the building, or even on a different day, and finally, return to the physician to initiate treatment if required. Most physicians also rely on the radiology interpretation of the study, which brings in more significant variability as the quality is not always uniform.

POCUS allows the streamlining of all of these steps into one office visit for evaluation, diagnosis and treatment. When time is of the essence, as it is for the diagnosis and treatment of DDH, advancements that improve efficiency and reduce cost, such as POCUS, should be distributed widely for incorporation into practice.

Furthermore, POCUS provides specialized care in nontraditional settings, whether rural, resource-scarce, or, as recently found, settings affected by the COVID-19 pandemic. During the COVID-19 pandemic, POCUS allows patients access to adequate care and evaluation while avoiding the traditional hospital setting. Thus, POCUS prevents delays in care for immunocompromised patients or parents who may be anxious or afraid of going to the hospital. POCUS also facilitates providing orthopedic care to rural settings. Tran et al. reviewed POCUS applications in low- and middle-income countries. They found it particularly beneficial in high need, low resource areas allowing physicians to diagnose many pathologies and initiate care quickly [10]. Rural areas face many obstacles in access to care, including patient presentation delays, urgency underestimation, and lack of subspeciality and essential support services. POCUS helps address several of these [11]. Whether rural or less accessible, POCUS has recently been incorporated into telemedicine, further advancing its effects on providing care.

The popularity of telemedicine accelerated during the COVID-19 pandemic. POCUS has great potential within this avenue of health care. Murphy et al. published a prospective observational study on a virtual clinic for DDH in Ireland and found this virtual clinic decreased median wait times from referral to treatment, the numbers of overall visits, and the per-patient visit cost by $\$ 170$ with no adverse events. They found the gross savings throughout course of the study from all virtual visits amounted to $€ 588,804$ [12]. POCUS can further enhance telemedicine's ability to increase the scope of specialist opinions in less specialized hospitals [13]. If healthcare delivery systems could institute multimodal clinics, where virtual evaluations were an option, with POCUS to facilitate patients' ability to obtain adequate screening, the entire patient experience from diagnosis through treatment and follow-up for DDH could be transformed.

POCUS makes accessing health care more straightforward and less expensive for both patients and the system itself. Van Schaik et al. reviewed the potential savings in an emergency room setting for privately insured, out-of-network or uninsured, and Medicare/Medicaid patients. The estimated savings from POCUS for one 8-h shift were on average $\$ 1134.31, \$ 2826.31$, and $\$ 181.63$, respectively [14]. Thirdly, new literature highlights the time-saving aspects of POCUS. Villanueva et al. found each POCUS saved an average of $2 \mathrm{~h}$ per patient for follow-up of pediatric ureteropelvic junction obstruction after surgical intervention [15].

With current high-end portable devices having a price point of around $\$ 2000$, their usage has spread throughout hospitals, allowing operators to interpret images during patient visits [10]. There are many different POCUS systems; some have a single, one-time fee for the acquisition of 
the hardware which can export images to any PACS system; others have a lower cost for the hardware and subsequently a yearly fee for image processing and storage. This price point is relatively inexpensive such that it has become feasible for low- and middle-income countries to use POCUS to diagnose and manage diseases [10]. Studies across many fields, ranging from emergency medicine, general surgery, urology and primary care, have already demonstrated significant reductions in cost for providers [15-17]. Because of this, many medical center are incorporating POCUS training into their education. In 2016, Mengel-Jørgensen et al. surveyed 12 countries/regions, finding that most general practitioners received formal training of POCUS to implement in their practice and a few countries even reported integrating POCUS into the undergraduate medical education curriculum [18].

Specific to the costs associated with DDH, early diagnosis is crucial, but when diagnosed late, there are significantly more invasive and expensive procedures required for treatment. Carmichael et al. estimated 2008 costs for different treatments related to DDH. The following are average costs for the treatment episodes: $\$ 7133$ for closed reduction and casting, \$18,201 for failed closed reduction and open surgeries and finally $\$ 38,000$ when osteotomies are required [19]. Massive cost savings for all parties involved can be extrapolated from these estimates when considering the number of procedures that could be avoided if timely, accurate diagnostic and treatment care is provided with POCUS.

Our study, however, was not without limitations. First, a single institution was involved in the project in a large urban academic medical center. Moving forward, analysis of the specific time and cost savings across several systems could enhance the generalizability of our findings. Second, POCUS is user-dependent and can become technically more challenging as children develop. However, a recent publication highlighted the ease of acquiring the skill to perform POCUS for different providers [20]. Third, successful POCUS use in practice assumes the proficiency of the care provider. In our study, only one physician was performing the POCUS, so in future research we will expand to see how the exam length differs between physicians. Although this is not necessarily a limitation, it is an essential factor in this medium's application. As with all new technology, the user must be adequately trained in using and interpreting POCUS to incorporate it into their practice successfullywhich is true of any new technology. Additionally, further investigations need to be performed regarding comparing the quality of imaging and diagnostics of POCUS to traditional ultrasound. There is some literature available for abdominal and pleural US on the non-inferiority of POCUS, but little in the Orthopedic realm [21]. A study by Ghosh et al. has demonstrated higher positive and negative predictive values in diagnosing medial compartment knee injuries using
POCUS when compared to MRI [22]. Nonetheless, future research comparing POCUS to the traditional US is needed to understand further the role of POCUS in the care of DDH. Finally, there are no medicolegal implications of POCUS in this setting; as physicians we are using this as a tool in our clinical practice and are not providing formal reports. This may be different in other countries and should be investigated prior to incorporating it into one's practice.

Though not a true limitation, it is important to note we do not have objective, statistically significant patient-reported outcomes to add information as to how the use of POCUS and subsequent changes in timing and cost of the visits affected patient satisfaction. We do have anecdotal information regarding patient satisfaction, but as this paper's focus was an economic standpoint, HCA CAPS (Hospital Consumer Assessment of Healthcare Providers and Systems) scores were not collected and analyzed.

\section{Conclusion}

To our knowledge, this is the first study to analyze and report on the effects of POCUS on the cost and duration of a patient encounter for a primary diagnosis of DDH. Ultimately, our study demonstrated a statistically significant reduction in the duration and cost of a patient encounter for infants with DDH when they undergo POCUS rather than "formal" sonographic evaluation. This study illustrates the potential benefits for both patients and healthcare systems of implementing POCUS into physician practices.

\section{Compliance with Ethical Standards}

Conflict of Interest The authors declare that they have no conflict of interest.

Ethical Approval This article does not contain any studies with human or animal subjects performed by the any of the authors.

Informed Consent For this type of study, informed consent is not required.

\section{References}

1. Bialik, V., Bialik, G. M., Blazer, S., Sujov, P., Wiener, F., \& Berant, M. (1999). Developmental dysplasia of the hip: A new approach to incidence. Pediatrics, 103(1), 93-99.

2. Zhang, S., Doudoulakis, K. J., Khurwal, A., \& Sarraf, K. M. (2020). Developmental dysplasia of the hip. British Journal of Hospital Medicine (London), 81(7), 1-8.

3. Degnan, A. J., Hemingway, J., Otero, H. J., \& Hughes, D. R. (2021). Developmental hip dysplasia and hip ultrasound frequency in a large American payer database. Clinical Imaging, $76,213-216$. 
4. Woodacre, T., Ball, T., \& Cox, P. (2016). Epidemiology of developmental dysplasia of the hip within the UK: Refining the risk factors. Journal of Children's Orthopaedics, 10(6), 633-642.

5. Zhao, L., Ma, Q., Feng, X., Fan, L., Jiao, Q., Wang, S., et al. (2019). Screening for developmental dysplasia of the hip in infants in tibet identifies increased prevalence associated with altitude. Medical Science Monitor, 25, 5771-5775.

6. Wyles, C. C., Heidenreich, M. J., Jeng, J., Larson, D. R., Trousdale, R. T., \& Sierra, R. J. (2017). The John Charnley Award: Redefining the natural history of osteoarthritis in patients with hip dysplasia and impingement. Clinical Orthopaedics and Related Research, 475(2), 336-350.

7. Feng, W. J., Wang, H., Shen, C., Zhu, J. F., \& Chen, X. D. (2017). Severe cartilage degeneration in patients with developmental dysplasia of the hip. IUBMB Life, 69(3), 179-187.

8. Biedermann, R., \& Eastwood, D. M. (2018). Universal or selective ultrasound screening for developmental dysplasia of the hip? A discussion of the key issues. Journal of Children's Orthopaedics, 12(4), 296-301.

9. O'Beirne, J. G., Chlapoutakis, K., Alshryda, S., Aydingoz, U., Baumann, T., Casini, C., et al. (2019). International Interdisciplinary Consensus Meeting on the Evaluation of Developmental Dysplasia of the Hip. Ultraschall in der Medizin, 40(4), 454-464.

10 Tran, T. T., Hlaing, M., \& Krause, M. (2021). Point-of-care ultrasound: applications in low- and middle-income countries. Current Anesthesiology Reports. https://doi.org/10.1007/ s40140-020-00429-y

11. Lese, A., \& Sraj, S. (2019). Rural orthopedics: providing orthopedic care in rural communities. Orthopedics, 42(4), e350-e355.

12. Murphy, E. P., Fenelon, C., Kennedy, J. F., O'Sullivan, M. D., Noel, J., Kelly, P. M., et al. (2021). Establishing a virtual clinic for developmental dysplasia of the hip: A prospective study. Journal of Pediatric Orthopaedics. https://doi.org/10.1097/BPO.00000 00000001755

13. Carbone, M., Ferrari, V., Marconi, M., Piazza, R., Del Corso, A., Adami, D., et al. (2018). A tele-ultrasonographic platform to collect specialist second opinion in less specialized hospitals. Updates in Surgery, 70(3), 407-413.

14. Van Schaik, G. W. W., Van Schaik, K. D., \& Murphy, M. C. (2019). Point-of-Care Ultrasonography (POCUS) in a community emergency department: An analysis of decision making and cost savings associated with POCUS. Journal of Ultrasound in Medicine. https://doi.org/10.1002/jum.14910
15. Villanueva, J., Pifer, B., Colaco, M., Fox, J., Chaudhry, R., Schneck, F., et al. (2020). Point-of-care ultrasound is an accurate, time-saving, and cost-effective modality for post-operative imaging after pyeloplasty. Journal of Pediatric Urology. https://doi. org/10.1016/j.jpurol.2020.05.156

16. Bhagra, A., Tierney, D. M., Sekiguchi, H., \& Soni, N. J. (2016). Point-of-Care ultrasonography for primary care physicians and general internists. Mayo Clinic Proceedings. https://doi.org/10. 1016/j.mayocp.2016.08.023

17. Lentz, B., Fong, T., Rhyne, R., \& Risko, N. (2021). A systematic review of the cost-effectiveness of ultrasound in emergency care settings. Journal of Ultrasound. https://doi.org/10.1186/ s13089-021-00216-8

18. Mengel-Jørgensen, T., \& Jensen, M. B. (2016). Variation in the use of point-of-care ultrasound in general practice in various European countries. Results of a survey among experts. European Journal of General Practice, 22(4), 274-277.

19. Carmichael, K. D., Longo, A., Yngve, D., Hernandez, J. A., \& Swischuk, L. (2008). The use of ultrasound to determine timing of Pavlik harness discontinuation in treatment of developmental dysplasia of the hip. Orthopedics. https://doi.org/10.3928/01477 447-20110525-14

20. Jejurikar, N., Moscona-Mishy, L., Rubio, M., Cavallaro, R., \& Castañeda, P. (2021). What is the interobserver reliability of an ultrasound-enhanced physical examination of the hip in infants? A prospective study on the ease of acquiring skills to diagnose hip dysplasia. Clinical Orthopaedics and Related Research. https:// doi.org/10.1097/CORR.0000000000001863

21. Rykkje, A., Carlsen, J. F., \& Nielsen, M. B. (2019). Hand-held ultrasound devices compared with high-end ultrasound systems: A systematic review. Diagnostics (Basel), 9(2), 61.

22. Ghosh, N., Kruse, D., Subeh, M., Lahham, S., \& Fox, J. C. (2017). Comparing point-of-care-ultrasound (POCUS) to MRI for the diagnosis of medial compartment knee injuries. Journal of Medical Ultrasound, 25(3), 167-172.

Publisher's Note Springer Nature remains neutral with regard to jurisdictional claims in published maps and institutional affiliations. 